\title{
Short Communication: Potential Risk of Replication-Competent Virus in HIV-1 Env-Pseudotyped Virus Preparations
}

\author{
Miroslawa Bilska, Haili Tang, and David C. Montefiori
}

\begin{abstract}
Env-pseudotyped viruses are valuable reagents for studies of HIV-1 neutralizing antibodies. It is often assumed that all pseudovirus particles are capable of only a single round of infection, making them a safe alternative to work with live HIV-1. In this study, we show that some Env-pseudotyped virus preparations give rise to low levels of replication-competent virus. These levels did not compromise results in the TZM-bl neutralization assay; however, their presence highlights a need to adhere to the same level of biosafety when working with Env-pseudotyped viruses that are required for work with replication competent HIV-1.
\end{abstract}

Keywords: HIV, viral fitness, HIV envelope glycoproteins, retroviral vectors, envelope

$\mathbf{S}^{\mathrm{n}}$ TUDIES OF NEUTRALIZING ANTIBODIES in HIV-1-infected individuals and in preclinical and clinical trials of candidate vaccines place a heavy reliance on the use of Envpseudotyped viruses. ${ }^{1,2}$ These pseudotyped viruses are produced by cotransfecting 293T or other suitable cell lines with an Env expression vector and an Env-defective backbone vector. One popular backbone vector, pSG3 $\Delta$ env, is a fulllength HIV-1 subtype B genome in plasmid pTZ19U that contains a four nucleotide insertion that inactivates the env gene (NIH AIDS Reagent Program, catalogue \#11051; contributed by Drs. John C. Kappes and Xiaoyun Wu). Because most of env is preserved in this backbone, potential exists for recombination with the cotransfected functional env gene to produce replication-competent virus (RCV). The presence of RCV could compromise the neutralization assay by representing an unintended viral target for neutralization. It would also highlight a safety concern for pseudoviruses that are presumed to be replication incompetent and therefore low risk. We tested a large number of assay stocks of HIV-1 Envpseudotyped viruses made with the SG3 $\Delta$ env backbone for the presence of RCV. In addition, we tested a subset of these Env-pseudotyped viruses made with HIV-1 subtype A backbone, Q23 $\Delta$ env, containing a 431 base pair deletion of the V1-C2 region of gp120. ${ }^{3}$ We also tested a small subset of Envpseudotyped viruses made with an alternate HIV-1 subtype B backbone, NL4-3.Luc. $\mathrm{R}^{-} \mathrm{E}^{-4}$. Both backbone vectors are available from the NIH AIDS Reagent Program (Q23 $\Delta$ env catalogue \#11548, contributed by Dr. Julie Overbaugh; NL4-3.Luc.R ${ }^{-} \mathrm{E}^{-}$

catalogue \#3418, contributed by Dr. Nathaniel Landau). All Env-pseudotyped viruses were prepared as described. ${ }^{5}$

Phytohemagglutinin-stimulated human peripheral blood mononuclear cells (PHA-PBMCs) were inoculated with undiluted Env pseudovirus preparations in 96-well roundbottom culture plates in the presence of IL-2. ${ }^{6}$ PHA-PBMCs were washed after 1 day to remove the virus inoculum and the medium was replaced. Fresh PHA-PBMCs were added on day 7. Cultures were incubated for 12-14 days with regular changing of the medium every 3 days. Culture fluids from days 7,10 , and the final day of incubation were tested for the presence of p24 Gag antigen (PerkinElmer Life Sciences, Inc., Boston, MA). Culture fluids on the final day of incubation were further tested for infectious virus by transferring $50 \mu \mathrm{l}$ to 96-well plates containing TZM-bl cells in a total volume of $250 \mu \mathrm{l} /$ well (this transfer was made before collecting culture fluid for p24 assay). Luciferase reporter activity was measured after two days of incubation as described. ${ }^{5}$ Samples were considered RCV positive if elevated levels of both p24 Gag antigen and relative luminescence units (RLU) were detected. All assays used the same pool of donor PBMC, which was prescreened and found to be negative for HIV-1.

As seen in Table 1, 17\% (20/117) of Env-pseudotyped virus preparations tested positive for RCV. Four independent preparations of the subtype B pseudovirus, SF162.LS, were positive when made with the SG3 $\Delta$ Env backbone. One of two preparations of this pseudovirus was positive when made with the subtype A backbone, Q23 $\Delta$ env, whereas two preparations made

Department of Surgery, Duke University Medical Center, Durham, North Carolina.

(C) Miroslawa Bilska, et al., 2017; Published by Mary Ann Liebert, Inc. This Open Access article is distributed under the terms of the Creative Commons Attribution Noncommercial License (http://creativecommons.org/licenses/by-nc/4.0/) which permits any noncommercial use, distribution, and reproduction in any medium, provided the original author(s) and the source are credited. 
Table 1. Test for Replication-Competent Virus in HIV-1 EnV-Pseudotyped Virus Preparations

\begin{tabular}{|c|c|c|c|c|c|c|}
\hline \multirow[b]{2}{*}{ Env-pseudotype } & \multirow[b]{2}{*}{ Backbone } & \multirow[b]{2}{*}{ Clade } & \multicolumn{3}{|c|}{$p 24(n g / m l)$} & \multirow{2}{*}{$\frac{R L U \text { in } T Z M-b l}{d 12-14 \text { PBMC supernatant }}$} \\
\hline & & & Day 7 & Day 10 & Day $12-14$ & \\
\hline Q168.a2 & Q23 & $\mathrm{AD}$ & 2.1 & 1.2 & 2.2 & 2061 \\
\hline Q168.a2 & Q23 3 env & $\mathrm{AD}$ & 1.7 & 0.1 & 0.1 & 1317 \\
\hline Q168.a2 & SG3 $\Delta$ Env & $\mathrm{AD}$ & 0.6 & 0.2 & 0.2 & 1889 \\
\hline Q23.17 & Q23 $\Delta$ env & $\mathbf{A}$ & 39.7 & 47.2 & 123 & 141010 \\
\hline Q23.17 & SG3 $\Delta$ Env & $\mathrm{A}$ & 5.4 & 0.2 & 0.1 & 1558 \\
\hline Q259.d2.17 & $\mathrm{Q} 23 \Delta \mathrm{env}$ & A & 3.5 & 1.9 & 3.6 & 1859 \\
\hline Q259.d2.17 & SG3 $\Delta$ Env & A & 1.8 & 0.5 & 0.5 & 2033 \\
\hline Q461.e2 & $\mathrm{Q} 23 \Delta \mathrm{env}$ & $\mathrm{AD}$ & 7.2 & 4.1 & 6.6 & 2615 \\
\hline Q461.e2 & SG3 $\Delta$ Env & A & 1.2 & 0.4 & 0.4 & 2054 \\
\hline Q769.d22 & Q23 $\Delta$ env & $\mathbf{A}$ & 7.8 & 4.3 & 8.2 & 31689 \\
\hline Q769.d22 & SG3 $\Delta$ Env & A & 3.2 & 0.1 & 0.1 & 1271 \\
\hline Q842.d12 & $\mathrm{Q} 23 \Delta \mathrm{env}$ & A & 3.5 & 1.6 & 3.1 & 1596 \\
\hline Q842.d12 & SG3 $\Delta$ Env & A & 0.7 & 0.1 & 0.1 & 1278 \\
\hline $235-47$ & SG3 $\Delta$ Env & $\mathrm{AG}$ & 1.7 & 0.1 & 0.1 & 1773 \\
\hline $252-7$ & SG3 $\Delta$ Env & $\mathrm{AG}$ & 2.6 & 0.2 & 0.1 & 1942 \\
\hline $263-8$ & SG3 $\Delta$ Env & $\mathrm{AG}$ & 1.3 & 0.1 & 0.1 & 1758 \\
\hline $271-11$ & SG3 $\Delta$ Env & $\mathrm{AG}$ & 1.4 & 0.1 & 0.1 & 1675 \\
\hline $\mathrm{T} 251-18$ & SG3 $\Delta$ Env & $\mathrm{AG}$ & 5.4 & 0.2 & 0.1 & 1736 \\
\hline $\mathrm{T} 255-34$ & SG3 $\Delta$ Env & $A G$ & 0.6 & 0.1 & 0.1 & 2243 \\
\hline $\mathrm{T} 257-31$ & SG3 $\Delta$ Env & $A G$ & 0.1 & 0.1 & 0.1 & 1519 \\
\hline T266-60 & SG3 $\Delta$ Env & $\mathrm{AG}$ & 0.8 & 0.1 & 0.1 & 1412 \\
\hline $\mathrm{T} 280-5$ & SG3 $\Delta$ Env & AG & 0.8 & 0.1 & 0.1 & 1584 \\
\hline 6101.1 & SG3 $\Delta$ Env & B & 3.4 & 0.5 & 2.2 & 33183 \\
\hline 6535.3 & $\mathrm{Q} 23 \Delta \mathrm{env}$ & $\mathrm{B}$ & 0.1 & 0.1 & 0.1 & 1628 \\
\hline 6535.3 & SG3 $\Delta$ Env & $\mathrm{B}$ & 0.3 & 0.1 & 0.1 & 1600 \\
\hline 62357.14.D3.4589 & SG3 $\Delta$ Env & B & 3.3 & 7.2 & 7.5 & 119276 \\
\hline 6240.08.TA5.4622 & SG3 3 Env & $\mathrm{B}$ & 1 & 0.4 & 0.3 & 2118 \\
\hline 6244.13.B5.4576 & SG3 $\Delta$ Env & $\mathbf{B}$ & 3.7 & 41.4 & 61.1 & 110315 \\
\hline 6535.3 ID & SG3 $\Delta$ Env & $\mathrm{B}$ & 0.1 & 0.1 & 0.1 & 2057 \\
\hline 700010040.C9.4520 & SG3 $\Delta$ Env & $\mathrm{B}$ & 2.2 & 0.4 & 0.4 & 2069 \\
\hline $9021-14 . B 2.4571$ & SG3 $\Delta$ Env & $\mathrm{B}$ & 3.4 & 0.9 & 0.8 & 2034 \\
\hline AC10.0.29 & SG3 $\Delta$ Env & $\mathrm{B}$ & 2.4 & 0.2 & 0.1 & 2422 \\
\hline $\mathrm{AC} 10.0 .29$ & SG3 $\Delta$ Env & B & 0.9 & 0.3 & 0.4 & 2062 \\
\hline AC10.0.29 & SG3 $\Delta$ Env & $\mathrm{B}$ & 0.4 & 0.2 & 0.6 & 1829 \\
\hline Bal.26 & SG3 $\Delta$ Env & $\mathrm{B}$ & 4.0 & 0.2 & 0.1 & 5586 \\
\hline BB-1006-11.C3.1601 & SG3 $\Delta$ Env & $\mathrm{B}$ & 0.4 & 0.2 & 0.2 & 2081 \\
\hline BB-1012-11.TC21 & SG3 $\Delta$ Env & $\mathrm{B}$ & 0.7 & 0.3 & 0.2 & 1952 \\
\hline BB-1054-07.TC4.1499 & SG3 $\Delta$ Env & $\mathbf{B}$ & 3.3 & 20.5 & 32 & 98849 \\
\hline BB-1056-10.TA11.1826 & SG3 $\Delta$ Env & $\mathrm{B}$ & 0.5 & 0.3 & 0.2 & 2107 \\
\hline BZ167.12 & SG3 $\Delta$ Env & $\mathbf{B}$ & 8.6 & 19.9 & 21.3 & 16897 \\
\hline CAAN5342.A2 & SG3 $\Delta$ Env & $\mathrm{B}$ & 1.1 & 0.4 & 0.5 & 2118 \\
\hline CAAN5342.A2 & SG3 $\Delta \Delta$ Env & $\mathrm{B}$ & 1.1 & 0.8 & 2.5 & 3349 \\
\hline CAAN5342.A2 & SG3 $\Delta$ Env & $\mathrm{B}$ & 0.4 & 0.2 & 0.5 & 1816 \\
\hline H022.7 & SG3 $\Delta$ Env & $\mathrm{B}$ & 3.2 & 0.1 & 3.3 & 9824 \\
\hline H030.7 (T2) & SG3 $\Delta$ Env & B & 2.2 & 0.1 & 0.1 & 1808 \\
\hline H035.18 & SG3 $\Delta$ Env & $\mathrm{B}$ & 2.2 & 0.1 & 0.1 & 1483 \\
\hline H077.31 (T1) & SG3 $\Delta$ Env & $\mathrm{B}$ & 2.0 & 0.1 & 0.1 & 1341 \\
\hline H079.2 (T1) & SG3 $\Delta$ Env & $\mathrm{B}$ & 0.3 & 0.1 & 0.1 & 1238 \\
\hline PRB926-04.A9.4237 & SG3 $\Delta$ Env & B & 3.3 & 29.3 & 27 & 119652 \\
\hline PVO.4 & Q23 3 env & $\mathrm{B}$ & 2.4 & 1.4 & 2.4 & 1795 \\
\hline PVO.4 & SG3 $\Delta$ Env & $\mathrm{B}$ & 0.8 & 0.3 & 0.3 & 2030 \\
\hline QH0692.42 & Q23 3 env & $\mathrm{B}$ & 5.4 & 2.9 & 5.1 & 1529 \\
\hline QH0692.42 & SG3 $\Delta$ Env & B & 18.1 & 42.2 & 91.9 & 187048 \\
\hline QH0692.42 & SG3 $\Delta$ Env & $\mathbf{B}$ & 6.0 & 142.5 & 198 & 92427 \\
\hline QH0692.42 & SG3 $\Delta$ Env & $\mathrm{B}$ & 2.2 & 0.7 & 0.8 & 1940 \\
\hline QH0692.42 & SG3 $\Delta$ Env & B & 2.1 & 2 & 3.1 & 10815 \\
\hline QH0692.42 & SG3 $\Delta$ Env & $\mathrm{B}$ & 3.5 & 2 & 3.3 & 1989 \\
\hline REJO4541.67 & SG3 $\Delta$ Env & $\mathrm{B}$ & 1.0 & 0.2 & 0.3 & 2000 \\
\hline REJO4541.67 & SG3 $\Delta$ Env & $\mathrm{B}$ & 1.9 & 1.3 & 2.1 & 1761 \\
\hline RHPA. 7 & SG3 $\Delta$ Env & $\mathrm{B}$ & 0.7 & 0.4 & 0.7 & 1664 \\
\hline RHPA4259.7 & SG3 $\Delta$ Env & $\mathrm{B}$ & 0.6 & 0.2 & 0.3 & 2028 \\
\hline SC05.8C11.2344 & SG3 $\Delta$ Env & $\mathrm{B}$ & 1.2 & 0.4 & 0.3 & 1988 \\
\hline
\end{tabular}


TABle 1. (CONTINUED)

\begin{tabular}{|c|c|c|c|c|c|c|}
\hline \multirow[b]{2}{*}{ Env-pseudotype } & \multirow[b]{2}{*}{ Backbone } & \multirow[b]{2}{*}{ Clade } & \multicolumn{3}{|c|}{$p 24(n g / m l)$} & \multirow{2}{*}{$\frac{R L U \text { in } T Z M-b l}{d 12-14 \text { PBMC supernatan }}$} \\
\hline & & & Day 7 & Day 10 & Day $12-14$ & \\
\hline SC422661.8 & $\mathrm{SG} 3 \Delta$ Env & B & 1.3 & 0.4 & 0.5 & 1923 \\
\hline SC422661.8 & SG3 $\Delta$ Env & B & 1.9 & 0.8 & 1.7 & 1280 \\
\hline SF162.LS & NL4-3.Luc.R ${ }^{-} \mathrm{E}^{-}$ & $\mathrm{B}$ & 0.4 & 0.3 & 0.5 & 2108 \\
\hline SF162.LS & NL4-3.Luc. $\mathrm{R}^{-} \mathrm{E}^{-}$ & B & 0.1 & 0.1 & 0.2 & 1066 \\
\hline SF162.LS & Q23 $\Delta$ env & B & 11.7 & 29.9 & 57.1 & 125593 \\
\hline SF162.LS & Q23 3 env & $\mathrm{B}$ & 1.2 & 0.7 & 0.5 & 1894 \\
\hline SF162.LS & SG3 $\Delta$ Env & B & 343.5 & 122.2 & 70.4 & 221969 \\
\hline SF162.LS & SG3 $\Delta$ Env & B & 64.7 & 266.1 & 343.3 & 132423 \\
\hline SF162.LS & SG3 $\Delta$ Env & B & 17.4 & 19.9 & 63.2 & 81844 \\
\hline SF162.LS & SG3 $\Delta$ Env & B & 4.5 & 38.4 & 132.2 & 197350 \\
\hline 89.6P.18 & NL4-3.Luc. R $\mathrm{E}^{-}$ & B & 0.1 & 0.1 & 0.1 & 1847 \\
\hline 89.6P.18 & NL4-3.Luc. ${ }^{-} \mathrm{E}^{-}$ & $\mathrm{B}$ & 0.01 & 0.1 & 0.1 & 1849 \\
\hline 89.6P.18 & Q23 2 env & $\mathrm{B}$ & 1.5 & 0.9 & 1.5 & 1932 \\
\hline SF162P3.5 & SG3AEnv & B & 2.1 & 4.4 & 8.1 & 109604 \\
\hline SF162P3.5 & SG3 $\Delta$ Env & $\mathrm{B}$ & 0.8 & 0.3 & 0.3 & 2082 \\
\hline SS1196.1 & SG3 $\Delta$ Env & $\mathrm{B}$ & 1.2 & 0.1 & 0.1 & 2892 \\
\hline THRO4156.18 & SG3 $\Delta$ Env & B & 23.6 & 45.6 & 58.3 & 94297 \\
\hline THRO4156.18 & SG3 $\Delta$ Env & $\mathrm{B}$ & 1.1 & 0.4 & 0.5 & 1878 \\
\hline THRO4156.18 & SG3 $\Delta$ Env & $\mathrm{B}$ & 1 & 0.6 & 1.1 & 1737 \\
\hline TRJO4551.58 & $\mathrm{SG} 3 \Delta \mathrm{Env}$ & $\mathrm{B}$ & 0.6 & 0.2 & 0.3 & 1763 \\
\hline TRJO4551.58 & SG3 $\Delta$ Env & $\mathrm{B}$ & 0.1 & 0.1 & 0.1 & 1890 \\
\hline TRO.11 & SG3 $\Delta$ Env & $\mathrm{B}$ & 0.6 & 0.2 & 0.3 & 2080 \\
\hline TRO.11 & SG3 $\Delta$ Env & $\mathrm{B}$ & 0.2 & 0.2 & 0.2 & 1797 \\
\hline WEAU-d15.410.787 & SG3 $\Delta$ Env & $\mathrm{B}$ & 2.4 & 0.5 & 0.5 & 2095 \\
\hline WITO4160.33 & Q23 2 env & $\mathrm{B}$ & 1.1 & 0.8 & 1.4 & 1826 \\
\hline WITO4160.33 & SG3 $\Delta$ Env & B & 0.3 & 0.2 & 0.2 & 1984 \\
\hline $\mathrm{CH} 038.12$ & SG3 $\Delta$ Env & $\mathrm{BC}$ & 1.4 & 0.3 & 0.4 & 8835 \\
\hline CH070.1 & SG3 $\Delta$ Env & $\mathrm{BC}$ & 2.1 & 0.1 & 0.1 & 1675 \\
\hline CH110.2 & $\mathrm{SG} 3 \Delta \mathrm{Env}$ & $\mathrm{BC}$ & 2.7 & 0.2 & 0.1 & 1644 \\
\hline CH115.12 & SG3 $\Delta$ Env & $\mathrm{BC}$ & 0.3 & 0.1 & 0.1 & 2036 \\
\hline CH119.10 & SG3 $\Delta$ Env & $\mathrm{BC}$ & 1.5 & 0.1 & 0.1 & 1657 \\
\hline CH181.12 & $\mathrm{SG} 3 \Delta \mathrm{Env}$ & $\mathrm{BC}$ & 2.4 & 0.1 & 0.1 & 1705 \\
\hline 97ZA012.12 & $\mathrm{Q} 23 \Delta \mathrm{env}$ & $\mathrm{C}$ & 0.7 & 0.4 & 0.8 & 1817 \\
\hline CAP210.2.00.E8 & SG3 $\Delta$ Env & $\mathrm{C}$ & 0.3 & 0.1 & 0.2 & 1935 \\
\hline CAP45.2.00.G3 & $\mathrm{SG} 3 \Delta \mathrm{Env}$ & $\mathrm{C}$ & 0.3 & 0.2 & 0.2 & 1993 \\
\hline Du151.2 & SG3 $\Delta$ Env & $\mathrm{C}$ & 4.5 & 0.2 & 0.1 & 1522 \\
\hline Du156.12 & SG3 $\Delta$ Env & $\mathrm{C}$ & 0.5 & 0.2 & 0.2 & 2008 \\
\hline DU172.17 & Q23 $\Delta$ env & $\mathrm{C}$ & 0.1 & 0.1 & 0 & 1747 \\
\hline Du172.17 & SG3 $\Delta$ Env & $\mathrm{C}$ & 0.8 & 0.1 & 0.1 & 1821 \\
\hline Du422.1 & SG3 $\Delta$ Env & $\mathrm{C}$ & 1.8 & 0.1 & 0.1 & 1099 \\
\hline HIV-0013095-2.11 & SG3 $\Delta$ Env & $\mathrm{C}$ & 0.6 & 0.1 & 0.1 & 1554 \\
\hline HIV-00836- 2.5 & SG3 $\Delta$ Env & $\mathrm{C}$ & 0.8 & 0.1 & 0.1 & 1303 \\
\hline HIV-16845-2.22 & SG3 $\Delta$ Env & $\mathrm{C}$ & 1.2 & 0.1 & 0.1 & 1594 \\
\hline HIV-25710-2.43 & SG3 $\Delta$ Env & $\mathrm{C}$ & 1.4 & 0.1 & 0.1 & 1631 \\
\hline HIV-25925-2.22 & $\mathrm{SG} 3 \Delta \mathrm{Env}$ & $\mathrm{C}$ & 2.4 & 0.2 & 0.1 & 1759 \\
\hline ZM106F.PB9 & $\mathrm{SG} 3 \Delta$ Env & $\mathrm{C}$ & 5.0 & 0.2 & 0.1 & 1566 \\
\hline ZM109F.PB4 & $\mathrm{SG} 3 \Delta \mathrm{Env}$ & $\mathrm{C}$ & 0.4 & 0.2 & 0.2 & 1994 \\
\hline ZM135M.PL10a & SG3 $\Delta$ Env & $\mathrm{C}$ & 0.2 & 0.1 & 0.1 & 2036 \\
\hline ZM197M.PB7 & SG3 $\Delta$ Env & $\mathrm{C}$ & 7.6 & 0.2 & 0.1 & 1413 \\
\hline ZM197M.PB7 & SG3 $\Delta$ Env & $\mathrm{C}$ & 0.5 & 0.2 & 0.2 & 1973 \\
\hline ZM214M.PL15 & SG3 $\Delta$ Env & $\mathrm{C}$ & 0.7 & 0.3 & 0.2 & 2074 \\
\hline ZM233M.PB6 & $\mathrm{Q} 23 \Delta \mathrm{env}$ & $\mathrm{C}$ & 0.8 & 0.6 & 1.2 & 1296 \\
\hline ZM233M.PB6 & SG3 $\Delta$ Env & $\mathrm{C}$ & 0.2 & 0.2 & 0.2 & 2057 \\
\hline ZM249M.PL1 & SG3 $\Delta$ Env & $\mathrm{C}$ & 0.4 & 0.2 & 0.2 & 1941 \\
\hline ZM53M.PB12 & SG3 $\Delta$ Env & $\mathbf{C}$ & 1.3 & 10.3 & 15.8 & 56045 \\
\hline
\end{tabular}

RLU, values $>9,000$ were considered positive and are bolded; p24 values $>3 \mathrm{ng} / \mathrm{ml}$ were considered positive and are bolded. Envpseudotyped viruses that tested positive for both RLU and p24 are also bolded.

PBMC, peripheral blood mononuclear cell; RLU, relative luminescence units. 
with the NL4-3.Luc. ${ }^{-} \mathrm{E}^{-}$backbone were both negative. Among six different preparations of subtype B pseudovirus QH0692.42 made with the SG3 $\Delta$ env backbone, three tested positive for $\mathrm{RCV}$. One of three different preparations of the subtype B pseudovirus THRO4156.18 made with SG3 $\Delta$ env backbone was positive. In other cases, multiple preparations of the same pseudoviruses made with the SG3 $\Delta$ env backbone were consistently negative (e.g., AC10.0.29, CAAN5342.A2, REJO4541 .67, SC422661.8, TRJO4551.58, TRO.11, and ZM197M.PB7 Envs). Thus, certain Envs appear to have a greater tendency to generate RCV than others. This tendency may also be related to the backbone vector. Thus, nonsubtype $B$ viruses made with the subtype B backbone SG3 $\Delta$ env were rarely RCV positive (1/41 cases positive, where the only positive was ZM53M.PB12, a subtype C Env). Among seven subtype A pseudoviruses made with the subtype A backbone Q23 $\Delta$ env, one tested positive and this single positive was an Env that was isogenic to the vector (Q23.17). Overall, of the 20 RCV-positive tests, only two occurred when the subtype of the Env and backbone were mismatched (SF162.LS made with Q23 $\Delta$ env; ZM53M.PB12 made with SG3 $\Delta$ env). These results indicate that the potential to generate RCV may be greater when using a backbone that is matched to the subtype of the Env clone. That being said, use of a nonsubtype-matched backbone vector is no guarantee that RCV will be avoided.

To confirm that recombination did in fact occur, we repeated a set of PBMC infection assays with three transfection stocks of Env pseudoviruses that tested positive for RCV and determined the Env sequences in the infected PBMC. These repeat tests were performed with the SG3 $\mathrm{EEnv}$ backbone pseudotyped with BZ167.12 and 62357.14.D3.4589 Envs and with the Q23 $\Delta$ env backbone pseudotyped with SF162.LS Env. All three transfection stocks of pseudoviruses once again tested positive for $\mathrm{p} 24 \mathrm{Gag}$ antigen after 10 days of PBMC infection.
Bulk gp160 sequencing of PBMC DNA was performed as described previously. ${ }^{7}$ Sequence analysis revealed the presence of recombinant gp160 genes in all three cases, where the defective region of the backbone gp160 was replaced with an overlapping region of the pseudotype gp160 (Fig. 1).

We next tested whether RCV impacted the outcome of neutralization assays performed in TZM-bl cells. Any impact would depend on where the RCV arose (i.e., during transfection or after infection) and how much RCV was present. Any RCV that arose after infection in TZM-bl cells would be of little consequence for an assay that is not incubated long enough to detect multiple rounds of infection. In contrast, if recombination occurred during transfection, the resulting virus would be a mixed population containing the intended Env and a recombinant Env containing elements of the backbone vector. In this latter case, levels of RCV that are sufficient to register in the assay might complicate the results by measuring neutralization of the RCV rather than solely the intended virus.

For our tests, we selected two separate stocks of SF162.LS and one stock of QH0692.42 that scored among the highest RCV values. These transfection stocks of Env-pseudotyped viruses that tested positive for RCV were assayed in parallel with a transfection stock of each Env-pseudotyped virus that tested negative for RCV. All Env-pseudotyped viruses were made with the SG3 $\Delta$ env backbone except for the RCVnegative stock of SF162.LS, which was made with the NL43.Luc. $\mathrm{R}^{-} \mathrm{E}^{-}$backbone. Assays were performed in TZM-bl cells ${ }^{5}$ using $13 \mathrm{HIV}-1$ plasma samples, soluble CD4, and the monoclonal antibodies IgG1b12, 2G12, 2F5, and 4E10. Equivalent neutralization results were obtained with all reagents regardless of RCV status ( $<3$-fold difference in ID50 neutralization titers between RCV positive and negative pairs of Env-pseudotyped viruses, data not shown). These results indicate that the level of RCV present in these Env-pseudotyped

\section{recombination Envelope region}

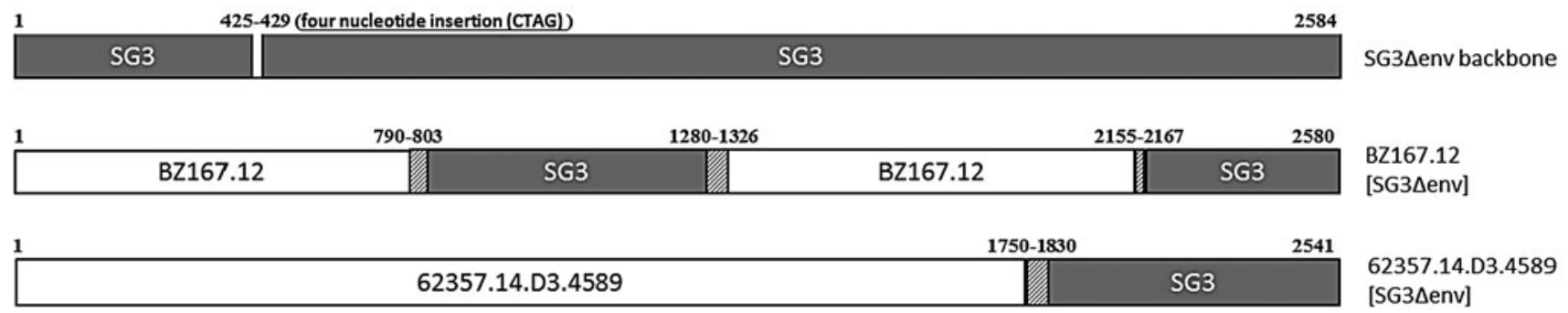

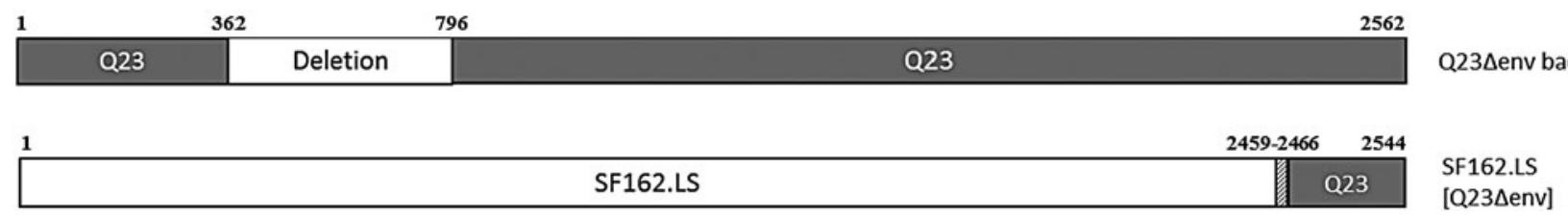

FIG. 1. Genetic recombination in replication-competent virus. Shown are the recombination events that occurred between backbone and pseudotyped $e n v$ genes detected in peripheral blood mononuclear cell DNA 10 days after infection with three different transfection stocks of Env-pseudotyped viruses. Backbone Env segments are shaded gray. Pseudotyped Env segments are not shaded. Nucleotide residues at the borders of mutations (backbone vectors) and regions of recombination are shown above each Env. 
viral preparations had no measurable effect on the TZM-bl neutralization assay.

Regardless of the lack of measurable impact of RCV on the results of neutralization assays, the real potential for RCV to be present at low levels in Env-pseudotyped virus preparations should raise awareness for laboratory personnel who may consider Env-pseudotyped viruses to be less of a risk and, therefore, require a lower level of biosafety than work with replication-competent HIV-1. We strongly recommend that all laboratory procedures with HIV-1 Env-pseudotyped viruses be conducted using the same level of biosafety that is required for work with replication competent HIV-1. At the very least, rigorous testing for RCV should be performed on all pseudovirus preparations before initiating work at a lower biosafety level.

\section{Acknowledgments}

The authors thank Dr. Richard Frothingham for recommending RCV testing of our Env-pseudotyped viruses. This work was supported by grants from the Bill \& Melinda Gates Foundation (Collaboration for AIDS Vaccine Discovery, Comprehensive Antibody Vaccine Immune Monitoring Consortium, \#1032144) and the U.S. National Institutes of Health (HHSN27201100016C).The funders had no role in the study design, data collection and analysis, the decision to publish, or drafting of the article.

\section{Author Disclosure Statement}

No competing financial interests exist.

\section{References}

1. Mascola JR, D'Souza P, Gilbert P, Hahn B, Haigwood NL, Morris L, et al.: Recommendations for the design and use of standard virus panels to assess the neutralizing antibody response elicited by candidate human immunodeficiency virus type 1 vaccines. J Virol 2005;79:10103-10107.

2. DeCamp A, Hraber P, Bailer RT, Seaman MS, Ochsenbauer C, Kappes J, et al.: Global panel of HIV-1 Env reference strains for standardized assessments of vaccine-elicited neutralizing antibodies. J Virol 2014;88:2489-2507.

3. Long EM, Rainwater SM, Lavreys L, Mandaliya K, Overbaugh J: HIV type 1 variants transmitted to women in Kenya require the CCR5 coreceptor for entry, regardless of the genetic complexity of the infecting virus. AIDS Res Hum Retroviruses 20002;18:567-576.

4. Connor RI, Chen BK, Choe S, Landau NR: Vpr is required for efficient replication of human immunodeficiency virus type-1 in mononuclear phagocytes. Virology 1995;206:935944.

5. Montefiori DC: Measuring HIV neutralization in a luciferase reporter gene assay. Methods Mol Biol 2009;485:395-405.

6. Bures R, Gaitan A, Zhu T, Graziosi C, McGrath K, Tartaglia $\mathrm{J}$, et al.: Immunization with recombinant canarypox vectors expressing membrane-anchored gp120 followed by gp160 protein boosting fails to generate antibodies that neutralize R5 primary isolates of human immunodeficiency virus type 1. AIDS Res Hum Retroviruses 2000;16:2019-2035.

7. Li M, Gao F, Mascola JR, Stamatatos L, Polonis VR, Koutsoukos M, et al.: Human immunodeficiency virus type 1 env clones from acute and early subtype B infections for standardized assessments of vaccine-elicited neutralizing antibodies. J Virol 2005;79:10108-10125.

Address correspondence to: David C. Montefiori Department of Surgery Duke University Medical Center Box 2926

Durham, NC 27710

E-mail: david.montefiori@duke.edu 